\title{
Simulação realística no ensino de segurança do paciente: relato de experiência
}

Realistic simulation in patient safety education: experience report

Simulación realística en la enseñanza de seguridad del paciente: relato de experiencia

\author{
Tânia Solange Bosi de Souza MagnagoI, Jaqueline Scalabrin da Silva ${ }^{\mathrm{II}}$, Taís Carpes Lanes ${ }^{\mathrm{III}}$ \\ Juliana Dal OngaroIV ${ }^{\mathrm{IV}}$ Emanuelli Mancio Ferreira da Luz ${ }^{\mathrm{v}}$, Patrícia Tuchtenhagen ${ }^{\mathrm{VI}}$ \\ Rafaela Andolhe ${ }^{\mathrm{VII}}$
}

\begin{abstract}
Resumo: Objetivo: relatar a vivência de ensino-aprendizagem de segurança do paciente com estudantes de um Curso de Graduação em Enfermagem. Método: relato de experiência, desenvolvido no ano de 2017, a partir de atividades teórico-práticas na disciplina "A enfermagem e a segurança do paciente na atenção à saúde”. Utilizou-se como estratégia metodológica a simulação realística, realizada no Laboratório de Habilidades, com estudantes do terceiro semestre de um Curso de Graduação em Enfermagem, de uma Universidade Pública Federal. Resultados: foram realizadas duas simulações no primeiro semestre letivo de 2017. A primeira referente aos protocolos de Higienização das Mãos e Identificação Correta do Paciente; a segunda abordou o protocolo de Cirurgia Segura e dispositivos intravenosos, cateteres e sondas. Pontos fortes e pontos frágeis foram discutidos, oportunizando fortalecer conhecimentos, habilidades e atitudes dos participantes. Conclusões: a vivência dos estudantes sobre o uso da simulação realística foi positiva, possibilitando maior confiança baseada na experiência vivenciada.
\end{abstract}

Descritores: Educação em Enfermagem; Treinamento por simulação; Segurança do paciente; Enfermagem

Abstract: Aim: To report the experience of teaching and learning in patient safety with students from an undergraduate Nursing Course. Method: experience report, developed in 2017, from theoretical and practical activities. The methodological strategy used was the realistic simulation, performed at the Skills Laboratory, with

\footnotetext{
I Enfermeira. Professora. Doutora em Enfermagem. Universidade Federal de Santa Maria. Santa Maria, RS, Brasil. E-mail: tmagnago@terra.com.br. ORCID: https://orcid.org/0000-0002-5308-1604

II Enfermeira. Mestre em Enfermagem. Universidade Federal de Santa Maria. Santa Maria, RS, Brasil. E-mail: jaquelinescalabrin@gmail.com. ORCID: https://orcid.org/0000-0001-8259-6992

III Enfermeira. Mestranda em Enfermagem. Universidade Federal de Santa Maria. Santa Maria, RS, Brasil. E-mail: taislanes_rock@hotmail.com. ORCID: https://orcid.org/0000-0001-9337-7875

IV Enfermeira. Mestranda em Enfermagem. Universidade Federal de Santa Maria. Santa Maria, RS, Brasil. E-mail: dongaro.ju@gmail.com. ORCID: https://orcid.org/0000-0003-0058-0132

v Enfermeira. Doutoranda em Enfermagem. Universidade Federal de Santa Maria. Santa Maria, RS, Brasil. E-mail: emanuelli_ferreira@hotmail.com. ORCID: https://orcid.org/0000-0002-7799-5232

VI Enfermeira. Mestre em Enfermagem. Universidade Federal de Santa Maria. Santa Maria, RS, Brasil. E-mail: patytuchtuch@yahoo.com.br. ORCID: https://orcid.org/0000-0001-8487-7149

VII Enfermeira. Professora. Doutora em Enfermagem. Universidade Federal de Santa Maria, RS, Brasil. E-mail: rafaela.andolhe1@gmail.com. ORCID: https://orcid.org/0000-0003-3000-8188
} 
third semester students of a Nursing Undergraduate Course, from a Federal Public University. Results: Two simulations were performed in the first semester of 2017, which addressed the protocols of Hand Hygiene, Correct Patient Identification, Safe Surgery and intravenous devices, catheters and probes. Afterwards, discussions was done to strengthen participants' knowledge and skills. Conclusions: the students' experience on the use of realistic simulation was positive, allowing greater confidence based on their experience

key words: Education Nursing; Simulation training; Patient safety; Nursing

Resumen: Objetivo: relatar la vivencia de enseñanza-aprendizaje de seguridad del paciente con estudiantes de un Curso de Pregrado en Enfermería. Método: relato de experiencia, desarrollado en 2017, a partir de actividades teóricas y prácticas. La estrategia metodológica utilizada fue la simulación realística, realizada en el Laboratorio de Habilidades, con estudiantes del tercer semestre de un Curso de Pregrado en Enfermería, de una Universidad Pública Federal. Resultados: Se realizaron dos simulaciones en el primer semestre lectivo de 2017, que abordaron los protocolos de Higiene de Manos, Identificación Correcta del Paciente, Cirugía Segura y dispositivos intravenosos, catéteres y sondas. Posteriormente, se realizaron discusiones para fortalecer el conocimiento y las habilidades de los participantes. Conclusiones: la vivencia de los estudiantes sobre el uso de simulación realística fue positiva, posibilitando una mayor confianza fundamentada en la experiencia vivida.

Descriptores: Educación en Enfermería; Entrenamiento Simulado; Seguridad del Paciente; Enfermería

\section{Introdução}

A segurança do paciente (SP) vem assumindo destaque nas últimas décadas, em razão da preocupação com as evidências da ocorrência de eventos adversos evitáveis durante a assistência prestada aos pacientes. Nesse contexto, a Organização Mundial da Saúde (OMS) lançou a Aliança Mundial para Segurança do Paciente. Nessa aliança, vários países firmaram o compromisso de adotar medidas de melhorias no atendimento aos pacientes com o propósito de aumentar a qualidade dos serviços de saúde prestados. ${ }^{1}$ Dentre essas atividades, destaca-se o documento que orienta a construção de currículos que englobem disciplinas voltadas à SP na formação dos profissionais da área da saúde. ${ }^{2}$

Nesse sentido, o Ministério da Saúde, por meio do Programa Nacional de Segurança do Paciente, reafirma a importância e a necessidade da inclusão do tema no ensino, do nível técnico ao superior. ${ }^{2}$ Assim, algumas instituições têm buscado inovações nas metodologias de ensino para abordar essa temática e têm incluído a simulação realística (SR). Este é um método de ensino que possibilita aos estudantes treinamento de habilidades técnicas e comportamentais para garantir a qualidade e desempenho da equipe no cuidado ao paciente. ${ }^{3}$ Por meio da simulação vários recursos 
3 | Magnago TSBS, Silva JS, Taís Carpes Lanes, Ongaro JD, Luz EMF, Tuchtenhagen P, Andolhe R

podem ser utilizados em laboratório para aproximar os estudantes de situações semelhantes à realidade com elementos reais, em cenas de alta fidelidade, antes de iniciarem suas atividades nos campos práticos e assim vivenciar o primeiro contato com o paciente. ${ }^{4}$

A SR objetiva aprimorar a educação, o treinamento, a avaliação de performance, o ensaio clínico e a pesquisa. Ela possibilita a aquisição de novos conhecimentos, conceitos, habilidades técnicas, tomadas de decisão, atitudes, comportamentos, trabalho em equipe e profissionalismo. ${ }^{5}$ Permite que os estudantes vivenciem situações cotidianas em um ambiente fictício e seguro, com intuito de promover o aprendizado e o senso crítico.

A oportunidade de os estudantes vivenciarem a prática assistencial por meio da SR contribui para reflexão de suas habilidades técnicas e comportamentais. Nesse contexto, estudo brasileiro identificou que $76,9 \%$ dos estudantes do curso de enfermagem de uma Universidade Federal relataram que a SR promoveu a aproximação dos discentes a casos clínicos reais e contribuiu para o raciocínio crítico. ${ }^{6}$

As atividades da SR devem ser planejadas e organizadas conforme os conteúdos teóricos, para que os estudantes tenham a oportunidade de adequação da prática à teoria com segurança, agilidade e confiança durante sua atuação em ambientes clínicos. ${ }^{6}$ Estudo Australiano verificou que $83 \%$ dos estudantes de enfermagem concordaram que o conhecimento e habilidades adquiridos durante a SR poderia auxiliá-los em futuras práticas assistenciais. ${ }^{7}$ Para tal, 93\% relataram melhor desenvolvimento do raciocínio clínico e tomada de decisão, e 77\% alegaram que a SR oportunizou colocar em prática o conhecimento teórico adquirido em sala de aula. ${ }^{7}$

Justifica-se este relato devido ao pioneirismo da SR com estudantes do curso de Graduação em Enfermagem da Instituição. A relevância na utilização da SR se dá na promoção do desenvolvimento de aprendizagens significativas, demonstrando eficácia na educação cognitiva e comportamental dos estudantes. ${ }^{5}$ Além disso, oportuniza o trabalho em equipe, fortalecendo o vínculo profissional. ${ }^{6}$ 
Simulação realística no ensino sobre segurança do paciente: relato de experiência| 4

Ainda, a utilização da metodologia participativa e realística representa uma estratégia positiva no ensino, auxiliando na aprendizagem por meio da prática de técnicas de enfermagem, aprimorando as habilidades e agregando as competências aos estudantes. ${ }^{6}$ Corroborando a isso, estudos científicos destacam que a utilização da SR favorece o aprendizado crítico e reflexivo, o aperfeiçoamento de habilidades técnicas e a autoconfiança dos estudantes durante a assistência prestada ao paciente..$^{8-10}$

Frente ao exposto, delimitou-se a questão norteadora: como a metodologia da simulação realística pode contribuir para o ensino-aprendizagem, de segurança do paciente, para estudantes de um Curso de Graduação em Enfermagem? Logo, objetiva-se relatar a vivência de ensino-aprendizagem de segurança do paciente com estudantes de um Curso de Graduação em Enfermagem.

\section{Método}

Trata-se de um relato de experiência desenvolvido por estudantes da pós-graduação no exercício da Docência Orientada, disciplina obrigatória de um Programa de Pós-Graduação stricto sensu em Enfermagem de uma Universidade Pública do Sul do Brasil. Foram realizadas duas SR, no primeiro semestre letivo de 2017, com a participação de 29 estudantes matriculados no terceiro semestre do Curso de Graduação em Enfermagem, na disciplina “A Enfermagem e a Segurança do Paciente na Atenção à Saúde”. Essa disciplina foi inserida no Projeto Pedagógico do Curso de Graduação, em 2016, após o processo de reformulação curricular, ofertada semestralmente, e possui dois créditos teóricos (30 horas).

O método de ensino com simulações realísticas foi iniciado, em 2017, sob a coordenação de dois docentes e a colaboração de mestrandos e doutorandos em docência orientada; também participaram enfermeiros, atuantes no laboratório do curso. As SR foram realizadas no Laboratório de Habilidades do Curso de Enfermagem. Os cenários foram organizados de forma que possibilitassem um atendimento seguro aos pacientes e acompanhantes, e que também 
5 | Magnago TSBS, Silva JS, Taís Carpes Lanes, Ongaro JD, Luz EMF, Tuchtenhagen P, Andolhe R

proporcionassem tranquilidade na execução das atividades, tanto dos estudantes em simulação, quanto daqueles que estavam no papel de observadores.

Para o planejamento, implementação e avaliação da SR, foram seguidas as seguintes estratégias: ${ }^{11}$

$>$ Escolha do tema: para a primeira SR foram contemplados os protocolos de higienização das mãos (HM) e identificação do paciente; e para a realização da segunda SR, utilizou-se o protocolo de cirurgia segura e o conteúdo sobre uso seguro de dispositivos intravenosos, cateteres e sondas. Os protocolos foram enviados para o e-mail da turma, com uma semana de antecedência à SR, para leitura prévia.

Definição dos objetivos a serem alcançados com a simulação: propiciar um ambiente simulado de uma cena semelhante ao ambiente hospitalar; estimular os estudantes a identificarem potencialidades e fragilidades, refletindo sobre seus conhecimentos, habilidades e atitudes adquiridos na leitura prévia; ressignificar a aprendizagem, construindo novos saberes;

$>$ Elaboração dos casos clínicos, roteiro de execução, checklist de acompanhamento e avaliação;

Organização do cenário: a partir dos casos, o cenário foi montado e testado previamente pelo professor e demais colaboradores envolvidos nas cenas (enfermeiros, mestrandos e doutorandos). Os papéis de paciente e acompanhante foram desenvolvidos pelos mestrandos e doutorandos;

$>$ Organização da simulação: os estudantes foram selecionados, mediante sorteio, no dia da SR para atuarem como "atores” da cena. Os estudantes não contemplados nos sorteios assumiram a posição de observadores. Fez-se filmagem da simulação;

> Método de discussão: realização do debriefing após simulação e feedback.

O debriefing compreende o ensino e a reflexão com esclarecimentos relacionados às atividades realizadas nas simulações, de modo que fossem retomados os fatos positivos e aqueles que deveriam ser melhorados noutra prática experimental. ${ }^{12}$ Já no feedback, o docente 
Simulação realística no ensino sobre segurança do paciente: relato de experiência| 6

tem a possibilidade de fornecer informações para os alunos, conduzindo-os nas atividades e, assim, reforçando informações para melhorar a aprendizagem das habilidades. ${ }^{12}$ Em ambos os momentos, o professor tem a possibilidade de associar a teoria à prática e dar a oportunidade ao aluno de verbalizar sobre a vivência na simulação. ${ }^{12}$

Com intuito de assimilar melhor o cenário da simulação, os estudantes em cena receberam apoio/orientações tanto por meio de informações verbais do professor/facilitador, como das falas dos pacientes e acompanhantes; dos registros nos prontuários e nas placas de identificação dos pacientes, que ficavam acima da cabeceira dos leitos. Essas informações eram disponibilizadas de modo a não interferir na atitude/decisão do estudante durante a simulação.

As orientações sobre o cenário e casos clínicos, o desenvolvimento das simulações, o debriefing e o feedback tiveram duração de duas horas para cada episódio de simulação. Justifica-se este tempo para a realização das SR devido ser a carga horária semanal disponível da disciplina, bem como esta mostrou-se eficaz para o desenvolvimento das etapas propostas, principalmente ao propiciar a atenção dos estudantes à SR. Os objetivos da atividade foram apresentados aos estudantes, no início do semestre, sendo que a cada aula eles autorizaram, de forma verbal, a filmagem das simulações. Destaca-se que as imagens foram utilizadas, somente em sala de aula, para fundamentar o debriefing e o feedback.

\section{Resultados}

Para construção dos cenários e avaliação da SR, o professor forneceu um roteiro de ações para execução dos casos. A partir disso, os colaboradores (enfermeiros, mestrandos e doutorandos) auxiliaram na elaboração e montagem dos casos clínicos e cenários; e nas filmagens das cenas. Também atuaram no papel de pacientes, familiares/acompanhantes e profissionais da área da saúde. 
7 | Magnago TSBS, Silva JS, Taís Carpes Lanes, Ongaro JD, Luz EMF, Tuchtenhagen P, Andolhe R

No dia da SR, foram sorteados dois estudantes para participar como "atores"; os demais estudantes da turma fizeram o papel de observadores da cena. Para isso, receberam um roteiro de acompanhamento (checklist), contendo informações sobre as ações e etapas que deveriam ser desenvolvidas pelos estudantes que estavam atuando na SR. No checklist, era assinalado sim ou não aos itens, referente às práticas descritas nos protocolos explorados. As atividades desenvolvidas na SR foram filmadas e reproduzidas aos estudantes, proporcionando a visualização e discussão dos pontos fortes e frágeis sobre a temática em pauta, durante o debriefing e o feedback.

a) As simulações

Na primeira simulação, os estudantes deveriam atentar para o protocolo de identificação correta do paciente e para a prática de HM em seus cinco momentos: antes do contato com o paciente, antes da realização de procedimento asséptico, após risco de exposição a fluidos corporais, após contato com o paciente e em áreas próximas ao paciente. ${ }^{1}$ Neste caso, foi realizada a simulação com duas pacientes que possuíam nomes semelhantes e estavam internadas na mesma enfermaria. $\mathrm{Na}$ cena, os estudantes deveriam atentar aos procedimentos de verificação de sinais vitais, contagem do gotejamento da solução endovenosa, administração de antitérmico por via oral e encaminhamento de uma das pacientes para o exame de radiografia. Para cada procedimento realizado, era esperado que o estudante fizesse a HM e a conferência da identificação do paciente, conforme preconizado nos protocolos.

A segunda simulação contemplou o protocolo de cirurgia segura e uso seguro de dispositivos intravenosos, cateteres e sondas. Nesta, foram criados dois ambientes: o de unidade de internação e o de centro cirúrgico, nos quais os estudantes deveriam atentar para o preenchimento correto do checklist de cirurgia segura ${ }^{13-14}$ e observar o uso de dispositivos intravenosos, cateteres e sondas. 
Simulação realística no ensino sobre segurança do paciente: relato de experiência| 8

O checklist de cirurgia segura corresponde a uma lista de checagem que começa na unidade de internação, segue no recebimento do paciente no centro cirúrgico, na sala de operação e é finalizada após o término do procedimento no centro cirúrgico. Para verificar a atenção dos estudantes em cena, foram colocados alguns fatores de risco como: a) unidade de internação: marcação cirúrgica em membro errado, paciente sem pulseira de identificação; b) Centro Cirúrgico: equipe de cirurgia dispersa durante a conferência do checklist, profissionais com adornos e paciente com sonda vesical clampeada e, uma pinça caída no campo cirúrgico.

No que tange ao protocolo de cirurgia segura (aplicação do checklist) e o uso seguro de dispositivos intravenosos, cateteres e sondas, a conferência foi de forma verbal e manuscrita, conforme é aplicado na instituição de ensino que os estudantes atuam. Durante a SR na unidade de internação, o estudante em cena identificou o erro de marcação, mas ao remarcar, não retirou a marcação equivocada. Identificou e retirou os adornos, mas não percebeu que o paciente estava sem pulseira de identificação.

$\mathrm{Na}$ recepção do paciente no centro cirúrgico, o checklist foi preenchido adequadamente pelo estudante, que fez a pulseira de identificação e desmarcou o lado errado. Na sala de cirurgia, o estudante teve dificuldade de solicitar a atenção de alguns profissionais para o preenchimento do checklist de cirurgia segura e à retirada dos adornos. Quanto ao clampeamento da sonda vesical, no momento em que o paciente referiu dor em baixo ventre, o mesmo foi identificado e corrigido pelo estudante. $\mathrm{Na}$ fase "antes do paciente sair da sala de operação" foi realizada a contagem dos instrumentais e o estudante apontou a falta de uma pinça, a qual foi encontrada no campo cirúrgico.

Quanto à identificação correta do paciente, observou-se que os estudantes não verificaram as pulseiras antes de realizar os procedimentos propostos. A identificação foi realizada pela placa de localização, presente na cabeceira do leito, que continha o nome do paciente, data de nascimento, registro e riscos/alergias. Positivamente, os estudantes perceberam que os pacientes possuíam nomes semelhantes e que um deles apresentava alergia a 
9 | Magnago TSBS, Silva JS, Taís Carpes Lanes, Ongaro JD, Luz EMF, Tuchtenhagen P, Andolhe R

dipirona. Dessa maneira, evitou-se um erro de administração de medicamento. Referente à HM, observou-se que o procedimento foi realizado somente no início da atividade e não foram seguidos os cinco momentos preconizados no protocolo.

Durante as simulações, os estudantes observadores foram colocados em pontos estratégicos da sala para que não atrapalhassem ou intimidassem os estudantes em cena. Assim, puderam observar e preencher o checklist das atividades que estavam sendo desenvolvidas.

b) O debriefing e feedback

Após o término das simulações, realizou-se o debriefing, etapa na qual os estudantes atuantes em cena e os observadores puderam verbalizar os sentimentos vivenciados e refletir sobre a experiência. Ainda, oportunizou espaço para elencar as fragilidades e as potencialidades das ações realizadas durante a assistência prestada na simulação. Já no feedback, o docente por meio das reproduções das filmagens e da revisão dos checklist, oportunizou mixagem da teoria e prática assistencial, aos estudantes, docentes, mestrandos, doutorandos e enfermeiros do laboratório.

Os estudantes se depararam com a importância do conhecimento prévio e a atenção no cuidado, não só para as simulações como também para sua vida acadêmica e profissional. Compreenderam que os erros/falhas cometidos na prática assistencial podem ocasionar agravos à saúde do paciente. Por isso, a importância de simular práticas, em ambientes de laboratório, auxilia no desenvolvimento de habilidades e propicia segurança durante o processo de cuidado aos pacientes. O debriefing, realizado nesse momento, serviu para uma autorreflexão dos participantes. Estes verbalizaram os sentimentos em relação à vivência na SR, expuseram suas dúvidas, sugeriram readequações, identificaram as fragilidades, bem como os pontos positivos. Nesse contexto, os conceitos apreendidos em aula e os conhecimentos adquiridos, após a leitura dos protocolos, puderam ser revistos para subsidiar a futura prática profissional.

Para a primeira simulação, poucos estudantes haviam realizado a leitura prévia dos materiais e protocolos, o que dificultou a realização das atividades propostas. No entanto, para a 
Simulação realística no ensino sobre segurança do paciente: relato de experiência| 10

segunda simulação, os estudantes já estavam mais atentos e preparados, realizaram a leitura prévia dos materiais encaminhados, demonstrando maior autonomia e conhecimento no que estavam fazendo, tanto na atuação como nas observações. Apresentaram-se mais dinâmicos e atentos durante a atividade de simulação e no debriefing. As discussões foram mais consistentes, tendo sido apresentados argumentos válidos após a visualização, nas gravações, dos erros e acertos, bem como um maior domínio e segurança sobre suas ações na execução da simulação.

Os estudantes obtiveram adesão e mostraram interesse nas atividades. Sugeriram a ampliação das atividades de SR para as demais disciplinas do Curso de Graduação em Enfermagem, pois vivenciaram uma maior compreensão dos conteúdos ao atuarem nas cenas e assistir aos colegas em atuação. A realização do debriefing e do feedback após as simulações significou uma riqueza de conteúdo, devido à atenta avaliação realizada pelos estudantes/observadores ao preencher o checklist da cena.

$\mathrm{Na}$ avaliação da disciplina, realizada ao final do semestre, os estudantes relataram que a experiência com a SR foi excelente, reforçando o aprendizado, possibilitando um maior entendimento da dinâmica assistencial, da atuação em equipe e da apreensão do conteúdo abordado em aula. Expressaram que, durante as simulações, o nervosismo, a insegurança e a ansiedade foram semelhantes ao vivenciar a prática real, pois as cenas eram elaboradas em um cenário próximo da realidade assistencial.

\section{Discussão}

A importância da simulação se dá em sua aptidão de proporcionar experiências de todo o processo educativo e oferecer aos estudantes oportunidades para repetição, reconhecimento de padrões e tomada de decisão. $\mathrm{Na} \mathrm{SR}$, a fidelidade consta em criar um cenário com maior aproximação possível da realidade de acordo com a proposta. ${ }^{12}$ Neste intuito, a resolução dos 
11 | Magnago TSBS, Silva JS, Taís Carpes Lanes, Ongaro JD, Luz EMF, Tuchtenhagen P, Andolhe R

problemas, durante a simulação, acontece de acordo com o aprendizado do estudante e conforme o conteúdo fornecido previamente, de modo que ele possa se sentir capaz de resolvê-los. ${ }^{15}$

Inicialmente, os estudantes estavam apreensivos devido a não realização da leitura dos protocolos enviados, o que ocasionou insegurança no manejo do paciente e foi uma barreira inicial para a realização da SR. No entanto, no que se refere às habilidades, observou-se que na segunda SR os estudantes apresentaram maior autonomia, destreza manual e empoderamento durante a atuação.

Assim, evidencia-se que a SR desenvolve habilidades e competências aliadas ao processo reflexivo, crítico e transformador do discente, bem como o aprimoramento de habilidades e competências. ${ }^{16-17}$ Equipara-se com os resultados da SR, realizada com estudantes de medicina, que constatou o desenvolvimento de habilidades no decorrer das simulações, sendo estas a comunicação, liderança, tomada de decisão, trabalho em equipe e relacionamento com o paciente. ${ }^{18}$

Essa maturidade, no decorrer da disciplina, traz a reflexão sobre a importância da utilização da SR como metodologia de ensino efetiva e inovadora. A SR surgiu como estratégia de ensino-aprendizagem para o docente, pois auxilia na construção de experiências que necessitam a reflexão do discente. Além disso, oportuniza que ele adquira a capacidade de conduzir seu processo de formação e de aprendizagem, por meio da vivência de práticas clínicas em um ambiente seguro e próximo da realidade assistencial. ${ }^{4,19}$

Quanto aos sentimentos vivenciados pelos estudantes atuantes, vem ao encontro de um estudo realizado no Distrito Federal que descreve que os estudantes de Graduação em Enfermagem vivenciaram sentimentos como ansiedade e insegurança, os quais podem estar presentes durante a prática assistencial em cenários hospitalares. Ainda, nessa linha de pensamento, a utilização da SR, como método de ensino-aprendizagem, mostrou-se promissora e eficiente. Com isso, recomenda-se a incorporação da SR no sistema educacional multiprofissional, visto que fortalece o conhecimento técnico-científico dos envolvidos, proporciona maior segurança na atuação, contribuindo no fortalecimento da prática profissional. ${ }^{10,16}$ 
Simulação realística no ensino sobre segurança do paciente: relato de experiência| 12

Neste intuito, a utilização da SR na formação dos profissionais da área da saúde contempla o aprimoramento de habilidades em um ambiente no qual se permitem erros e que estes colaboram para o crescimento profissional, sem colocar em risco a SP. ${ }^{20}$ Nas simulações, os alunos ressaltaram a importância da autoavaliação nas atividades desenvolvidas, pois é possível a reflexão quanto aos erros e também uma melhor compreensão dos conteúdos. A estratégia debriefing é considerada como uma das etapas mais importantes na SR, pois o professor/facilitador conduz o aluno a uma reflexão da experiência, diante dos atos da simulação, auxiliando na percepção de sua atuação, na tomada de decisão e na sua competência diante do cenário realizado. ${ }^{12}$

Foram observados na SR o aprendizado, a fixação de conteúdo, o aumento do interesse pela disciplina e aproximação com a prática. E em concordância com a literatura, na qual avaliou a percepção de 37 estudantes de enfermagem, identificou que a SR proporciona uma vivência prévia da prática (23,9\%); permite fixar os conhecimentos adquiridos em sala de aula $(21,7 \%)$ e que contribui para relacionar a teoria e a prática $(15,2 \%) .{ }^{21}$

Estudantes de enfermagem descrevem a SR como estratégia que possibilita o melhor ensino-aprendizagem e sugerem sua extensão para todas as disciplinas aliadas às aulas teóricas. ${ }^{6}$ Além disso, a utilização da metodologia de SR aproxima os estudantes à realidade da prática assistencial, com vistas às trocas de experiências e no desempenho de habilidades práticas associadas a reflexão teórica, quando comparado ao método tradicional de ensino. ${ }^{22-23}$

\section{Conclusões}

A vivência de ensino-aprendizagem, na $S R$, sobre segurança do paciente, mostrou-se positiva no contexto dos estudantes, bem como para os docentes da disciplina e discentes da pós-graduação em docência orientada. Essa experiência motivou os envolvidos a expandir e enriquecer o ensino por meio deste método, pois a simulação é uma ferramenta efetiva e 
13 | Magnago TSBS, Silva JS, Taís Carpes Lanes, Ongaro JD, Luz EMF, Tuchtenhagen P, Andolhe R

inovadora que possibilita ao estudante se apresentar ao paciente com maior confiança, ou seja, baseada na experiência prévia vivenciada.

O uso da SR auxilia no gerenciamento de problemas da prática, flexibilidade, uso do conhecimento factual, pensamento crítico, interação com a equipe, tempo de resposta, habilidades de comunicação, planejamento, estratégia, decisões múltiplas e colaboração. Do mesmo modo, a metodologia de SR atua como ferramenta necessária para a formação dos futuros profissionais para os desafios de lidar com os pacientes nos diferentes ambientes de assistência, no que diz respeito às relações humanas com os assistidos, familiares e com a equipe multidisciplinar.

Este modo de ensino na disciplina se mostrou satisfatório, em que oportunizou uma aprendizagem de qualidade e preparação dos alunos para atividades práticas, oferecendo maior segurança e entendimento da realidade do ambiente real de trabalho. Durante a realização das simulações, o interesse, empenho e crescimento dos estudantes com as temáticas abordadas foram notórios. No contexto do que foi exposto, verifica-se a necessidade de novos estudos que abordem essa nova metodologia de ensino, que qualifica a formação dos futuros profissionais, subsidiando-os para uma prática crítica, criativa e segura.

\section{Referências}

1. Agência Nacional de Vigilância Sanitária (BR). Segurança do paciente em serviços de saúde: higienização das mãos. Brasília (DF): Ministério da Saúde; 2009.

2. Brasil. Ministério da Saúde. Portaria n. 529, de $1^{\text {o }}$ de abril de 2013. Institui o Programa Nacional de Segurança do Paciente (PNSP) [Internet]. 2013 [acesso em 2018 nov 14]. Disponível em: http://bvsms.saude.gov.br/bvs/saudelegis/gm/2013/prt0529_01_04_2013.html

3. Buxton M; Phillippi J; Collins MR. Simulation: a new approach to teaching ethics. J Midwifery Womens Health [Internet]. 2015 jan-fev [acesso em 2018 nov 14];60(1):70-4. Disponível em: https://onlinelibrary.wiley.com/doi/epdf/10.1111/jmwh.12185

4. Sanino GEC. O uso da simulação em enfermagem no curso técnico de enfermagem. J Health Inform [Internet]. $2012 \mathrm{dez}$ [acesso em $2017 \mathrm{dez}$ 10];4(N Esp):148-51. Disponível em: http://www.jhisbis.saude.ws/ojs-jhi/index.php/jhi-sbis/article/view/247/136 
Simulação realística no ensino sobre segurança do paciente: relato de experiência| 14

5. Melo MCB, Lui PMF, Magalhães AMPB, Gresta MM, Silva NLC, Brandão CFS. A simulação no ensino da graduação. In: Scalabrini Neto A, Fonseca AS, Brandão CFS. Simulação realística e habilidades na saúde. Rio de Janeiro (RJ): Atheneu; 2017. p. 23-29.

6. Valadares AFM, Magro MCS. Opinião dos estudantes de enfermagem sobre a simulação realística e o estágio curricular em cenário hospitalar. Acta Paul Enferm [Internet]. 2014 abr [acesso em 2018 dez 13]; 27(2):138-43. Disponível em: http://www.scielo.br/scielo.php?script=sci_arttext\&pid=S010321002014000200009\&lng=en http://dx.doi.org/10.1590/1982-0194201400025

7. Currie J, Kourouche S, Gordon C, Jorm C, West S. Mass casualty education for undergraduate nursing students in Australia. Nurse Educ Pract [Internet]. 2017 out [acesso em 2017 dez 01];(28):156-62. Disponível em: https://www.ncbi.nlm.nih.gov/pubmed/29101834

8. Bortolato-Major C, Arhur JP, Mattei AT, Mantovani MF, Felix JVC, Boostel R. Contribuições da simulação para estudantes de graduação em enfermagem. Rev Enferm UFPE On Line [Internet]. 2018 [acesso em $2017 \mathrm{dez}$ 01];12(6):1751-62. Disponível em: https://doi.org/10.5205/1981-8963-v12i6a230633p1751-1762-2018

9. Kaneko RMU, Lopes MHBM. Cenário em simulação realística em saúde: o que é relevante para a sua elaboração? Rev Esc Enferm USP [Internet]. 2019 [acesso em 2017 dez 01];53:e03453. Disponível em: http:// dx.doi.org/10.1590/S1980-220X2018015703453

10. Nascimento MS; Magro MCS. Simulação realística: método de melhoria de conhecimento e autoconfiança de estudantes de enfermagem na administração de medicamento. REME Rev Min Enferm [Internet]. 2018 mar [acesso em 2018 nov 25];22(e-1094):1-5. Disponível em: http://www.reme.org.br/artigo/detalhes/1232

11. Jeffries PR. Simulation in nursing education: from conceptualization to evaluation. New York: National League for Nursing; 2007.

12. Coutinho V, Martins JCA, Pereira MF, Mazzo A. Feedback e debriefing. In: Scalabrini Neto A, Fonseca AS, Brandão CFS. Simulação realística e habilidades na saúde. Rio de Janeiro (RJ): Atheneu; 2017. p. 115-25.

13. Organização Mundial da Saúde (OMS). Segundo desafio global para a segurança do paciente: cirurgias seguras salvam vidas [Internet]. Rio de Janeiro (RJ): Organização Pan-americana da Saúde, Ministério da Saúde, Agência Nacional de Vigilância Sanitária; 2009 [acesso em 2018 dez 15]. Disponível em: http://bvsms.saude.gov.br/bvs/publicacoes/seguranca_paciente_cirurgias_seguras_guia.pdf

14. Universidade Federal de Santa Maria (UFSM), Hospital Universitário de Santa Maria (HUSM). HUSM-UFSM-Hospital Universitário de Santa Maria [Internet]. 2016 [acesso em 2018 out 15]. Disponível em: http://www2.ebserh.gov.br/web/husm-ufsm

15. Mazzo A, Almeida RGS, Baptista RCN, Pedersoli CE, Girão FB, Martins JCA. Simulação: conceitos básicos. In: Scalabrini Neto A, Fonseca AS, Brandão CFS. Simulação realística e habilidades na saúde. Rio de Janeiro (RJ): Atheneu; 2017. p. 49-57. 
15 Magnago TSBS, Silva JS, Taís Carpes Lanes, Ongaro JD, Luz EMF, Tuchtenhagen P, Andolhe R

16. Magro MCS, Barreto DG, Silva KGN, Moreira SCR, Silva TS, Santos CE. Vivência prática de simulação realística no cuidado ao paciente crítico: relato de experiência. Rev Baiana Enferm [Internet]. 2012 maio-ago [acesso 2018 out 14];26(2):556-61. Disponível em: https://portalseer.ufba.br/index.php/enfermagem/article/view/6544/6360

17. Silva RC, Torres AAP, Valadão SR, Soares TMS. A simulação do cuidado como um cenário de aprendizagem em enfermagem. J Nurs Health. 2016;6(Supl.):164-72.

18. Brandão CFS, Collares CF, Marin HF. A simulação realística como ferramenta educacional para estudantes de medicina. Sci Med (Porto Alegre) [Internet]. 2014 dez [acesso em 2018 dez 01];24(2):187-92. Disponível em: http://revistaseletronicas.pucrs.br/ojs/index.php/scientiamedica/article/view/16189/11485

19. Oliveira SN, Prado ML, Kempfer SS. Utilização da simulação no ensino da enfermagem: revisão integrativa. Rev Min Enferm [Internet]. 2014 abr-jun [acesso em 2018 dez 01];18(2):487-95. Disponível em: http://www.dx.doi.org/10.5935/1415-2762.20140036

20. Abreu AG, Freitas JS, Berte M, Ogradowski KRP, Nestor A. O uso da simulação realística como metodologia de ensino e aprendizagem para as equipes de enfermagem de um hospital infanto-juvenil: relato de experiência. Ciênc Saúde (Porto Alegre). 2014;7(3):162-6.

21. Costa RRO, Medeiros SM, Martins JCA, Cossi MS, Araújo MS. Percepção de estudantes da graduação em enfermagem sobre a simulação realística. Cuid [Internet]. 2017 [acesso em 2017 dez 10];8(3): 1799-808. Disponível em: http://dx.doi.org/10.15649/cuidarte.v8i3.425

22. Monteiro AB, Peixoto JGP, Silva APSS, Caregnato RCA, Millão LF. Formação para segurança do paciente: uma experiência de integração entre alunos da graduação e pós-graduação. Rev Enferm UFSM [Internet]. 2018 jan-mar [acesso em 2018 dez 11];8(1):192-202. Disponível em: https://periodicos.ufsm.br/reufsm/article/view/23988/pdf

23. Rohrs RMS, Santos CF, Barbosa RS, Schulz RS, Carvalho MB. Impacto da metodologia de simulação realística na graduação de enfermagem. Rev Enferm UFPE On Line [Internet]. 2017 [acesso em $2018 \mathrm{dez}$ 11]:11(Supl 12):5269-74. Disponível em: https://doi.org/10.5205/1981-8963-v11i12a23005p5269-5274-2017

\section{Autor correspondente}

Tânia Solange Bosi de Souza Magnago

E-mail: tmagnago@terra.com.br

Endereço: Av. Roraima, 1000 - Camobi, Santa Maria - RS

CEP: $97105-900$ 
Simulação realística no ensino sobre segurança do paciente: relato de experiência| 16

\section{Contribuições de Autoria}

\section{1 - Tânia Solange Bosi de Souza Magnago}

Concepção e planejamento da disciplina e da simulação realística; análise e interpretação dos dados; Redação do artigo e revisão crítica relevante do conteúdo intelectual; Aprovação final da versão a ser publicada.

\section{2 - Jaqueline Scalabrin da Silva}

Análise e interpretação dos dados; Redação do artigo e revisão crítica relevante do conteúdo intelectual; Aprovação final da versão a ser publicada.

\section{3 - Taís Carpes Lanes}

Análise e interpretação dos dados; Redação do artigo e revisão crítica relevante do conteúdo intelectual; Aprovação final da versão a ser publicada.

\section{4 - Juliana Dal Ongaro}

Análise e interpretação dos dados; Redação do artigo e revisão crítica relevante do conteúdo intelectual; Aprovação final da versão a ser publicada.

\section{5 - Emanuelli Mancio Ferreira da Luz}

Análise e interpretação dos dados; Redação do artigo e revisão crítica relevante do conteúdo intelectual; Aprovação final da versão a ser publicada.

\section{6 - Patrícia Tuchtenhagen}

Análise e interpretação dos dados; Redação do artigo e revisão crítica relevante do conteúdo intelectual.

\section{7 - Rafaela Andolhe}

Concepção e planejamento da disciplina e da simulação realística; análise e interpretação dos dados; Redação do artigo e revisão crítica relevante do conteúdo intelectual; Aprovação final da versão a ser publicada.

\section{Como citar este artigo}

Magnago TSBS, Silva JS, Taís Carpes Lanes, Ongaro JD, Luz EMF, Tuchtenhagen P, Andolhe R. Simulação realística no ensino de segurança do paciente: relato de experiência. Rev. Enferm. UFSM. 2019 [Acesso em: Ano Mês Dia];vol.10 e13: 1-16. DOI:https://doi.org/10.5902/2179769236616 